Check for updates

Cite this: Phys. Chem. Chem. Phys., 2019, 21, 18769

Received 25th June 2019, Accepted 23rd July 2019 DOI: $10.1039 / c 9 c p 03581 \mathrm{~h}$ rsc.li/pccp

\title{
Orientational order in the splay nematic ground state $\uparrow$
}

\author{
Richard J. Mandle (D)*a and Alenka Mertelj (D) ${ }^{b}$
}

\begin{abstract}
Modulated nematic liquid crystal phases, which lack positional order but have some periodic variation in the direction of average orientation present in a classical nematic, have attracted significant interest. In the recently discovered splay nematic $\left(N_{S}\right)$ phase the average orientational order is augmented with a periodic splay deformation of orientation perpendicular to the director. We use X-ray scattering experiments to measure the orientational order parameters in the nematic $(N)$ and splay nematic $\left(N_{S}\right)$ phases of the liquid crystalline material RM734. The degree of orientational order is somewhat larger in the $\mathrm{N}_{\mathrm{S}}$ phase than in the preceding nematic and temperature dependent. We reconstruct the orientational distribution function and find it to be nematic-like in the $\mathrm{N}_{S}$ phase, indicating the change in orientation between neighbouring molecules due to the splay modulation is very small. A small splay angle implies that the splay modulation period is larger than the few tens of nanometers originally envisaged. The method described herein can be used to assist in unambiguous identification of the splay-nematic phase.
\end{abstract}

\section{Introduction}

Nematic liquid crystal phases, in which there is an average orientational order of the molecules but a lack of positional order, are the cornerstone of the display industry. New nematic ground states are rare, and in the 21st century the prediction and subsequent discovery of nematic phases with periodic modulation of their orientation has generated significant interest. $^{1-5}$ The most widely studied of these recently observed nematic variants is the twist-bend nematic $\left(\mathrm{N}_{\mathrm{TB}}\right)$, in which the average orientational direction precesses about a cone along the optic axis giving rise to a helical structure with a periodicity of a few molecular lengths. ${ }^{6-8}$

Some years ago we reported rod-shaped materials which exhibit two nematic phases separated by a weak first order phase transition. ${ }^{9}$ One of these materials (RM734, Fig. 1A) ${ }^{10}$ was studied in detail by Mertelj et al., who found the higher temperature phase to be a classical nematic phase, and the lower temperature nematic to be a nematic phase with a periodically splayed local director - a splay nematic phase $\left(\mathrm{N}_{\mathrm{S}}\right.$, Fig. 1C). ${ }^{11}$ The experimental observation of the splay nematic ground state satisfied an earlier prediction of such a phase. ${ }^{12}$ Chaturvedi and Kamien recently discussed how the $\mathrm{N}_{\mathrm{S}}$ is related to the splay-bend nematic phase $\left(\mathrm{N}_{\mathrm{SB}}\right)$ described by

\footnotetext{
${ }^{a}$ Department of Chemistry, University of York, York, YO10 5DD, UK.

E-mail: richard.mandle@york.ac.uk

${ }^{b}$ J. Stefan Institute, SI-1000 Ljubljana, Slovenia

$\dagger$ Electronic supplementary information (ESI) available. See DOI: 10.1039/ c9cp03581h
}

Dozov through exchange of the splay and bend elastic constants; ${ }^{1,13}$ the modulation vector is perpendicular to the director in the $\mathrm{N}_{\mathrm{S}}$ phase and parallel in the $\mathrm{N}_{\mathrm{SB}}$. It is possible that the 'MP' phase reported by Kikuchi et al. is another example of the splay nematic phase. ${ }^{14}$ Barbero and Lelidis recently discussed the possibility
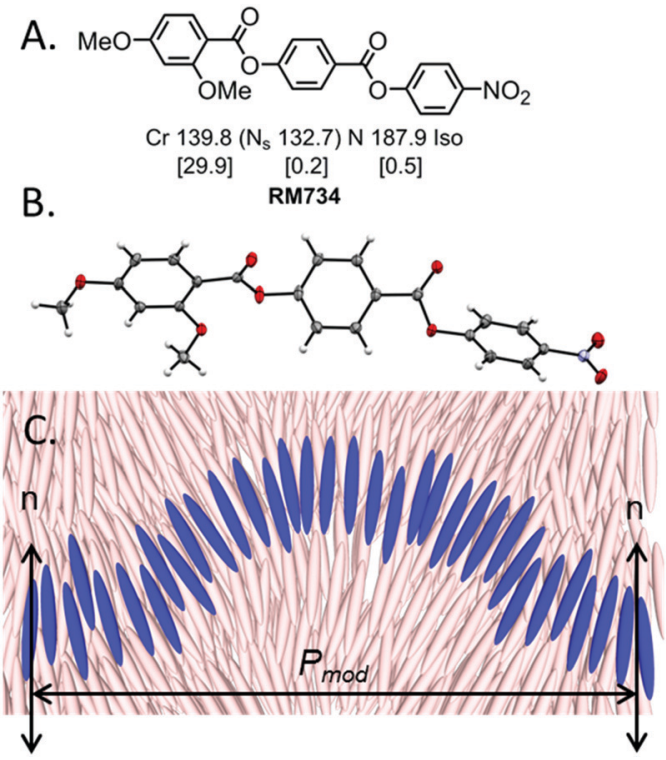

Fig. 1 (A) Molecular structure, transition temperatures $\left({ }^{\circ} \mathrm{C}\right.$ ) and associated enthalpies [kJ mol ${ }^{-1}$ ] of RM734; ${ }^{10}$ (B) ORTEP model of RM734 obtained by XRD as described in ref. 11 with thermal ellipsoids at $50 \%$ probability; (C) cartoon depiction of a single period $\left(P_{\text {mod }}\right)$ of the periodic splay deformation of the director $(n)$ in the $N_{S}$ phase. 
of periodic modulated splay-nematic phases that results from a negative value of $K_{11} \cdot{ }^{15}$ The proposed structure of the $\mathrm{N}_{\mathrm{S}}$ phase is polar, biaxial and antiferroelectric and therefore of significant interest for electrooptic devices. However, there is a need to better characterise the structure of this phase, and to devise methods for unambiguous identification.

For the twist-bend modulated nematic phase, X-ray scattering experiments can be used to measure the order parameters which can then be used to calculate the heliconical tilt angle. ${ }^{7,8}$ From the proposed model of the $\mathrm{N}_{\mathrm{S}}$ phase ${ }^{11}$ one can imagine that the periodicity of the splay-nematic phase is inversely related to the splay-angle between neighbouring molecules, i.e. a large periodic length scale leads to a small angle and vice versa. In the case of a small periodic length scale, this angle may be measurable by X-ray scattering experiments. However, its absence would support the idea that the periodic length scale of the $\mathrm{N}_{\mathrm{S}}$ phase is large, rather than being on the order of a few nanometers.

\section{Experimental}

Materials used in this study were prepared as described previously. ${ }^{10}$ The first five even orientational order parameters $\left(\left\langle P_{n}\right\rangle\right)$ of RM734 were determined by wide angle X-ray scattering (WAXS) using the method described in ref. 16 and 17 Samples were filled into $0.9 \mathrm{~mm}$ I.D. borosilicate glass capillary tubes purchased from Capillary Tube Supplies UK Ltd. Briefly, 2D WAXS patterns were recorded as a function of temperature in $3{ }^{\circ} \mathrm{C}$ intervals over a temperature range of $195{ }^{\circ} \mathrm{C}$ to $48{ }^{\circ} \mathrm{C}$ $\left(T / T_{\mathrm{N}-\text { Iso }}\right.$ range of 1.01 to 0.69$)$ with an exposure time of 300 seconds and a sample to detector distance of $121 \mathrm{~mm}$, allowing simultaneous recording of small and wide angle data. The 2D WAXS pattern obtained from an empty glass capillary was used as a background. Following background subtraction we performed azimuthal integration ( $1^{\circ}$ step size). Fitting according to eqn (1) allows the orientational order parameters to be determined; eqn (2) and (3) show this for $\left\langle P_{2}\right\rangle$ while details for higher order parameters are given in the ESI. $\dagger$

$$
\begin{gathered}
I(X)=\sum_{0}^{\infty} \frac{\pi}{2} f_{2 n} \frac{(2 n-1) ! !}{2^{n} n !} \cos ^{2 n} X \\
\left\langle\cos ^{2} \beta\right\rangle=\sum_{n=0}^{\infty} \frac{f_{2 n}}{2 n+3} / \sum_{n=0}^{\infty} \frac{f_{2 n}}{2 n+1} \\
\left\langle P_{2}\right\rangle=\frac{1}{2}\left(3\left\langle\cos ^{2} \beta\right\rangle-1\right)
\end{gathered}
$$

Form factors and scattering intensities were calculated from atomic coordinates using the ATSAS 2.8 software package. ${ }^{18-20}$

\section{Results and discussion}

As discussed in an earlier paper, when studied by X-ray scattering experiments the wide-angle peak RM734 (and related materials) is found over a particularly large angular range and

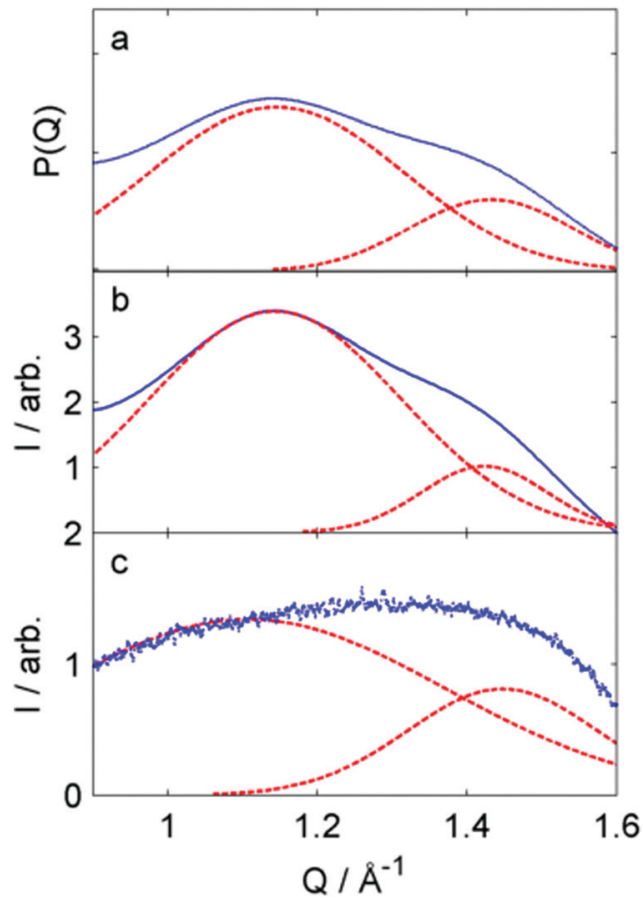

Fig. 2 (a) Plot of the calculated molecular form factor $(P(Q))$ of RM734. (b) Plot of the calculated wide angle scattered intensity of RM734. (c) Plot of experimentally measured scattered intensity versus $Q\left(\AA^{-1}\right)$ for the wideangle peak(s) of RM734 in the isotropic liquid at $190{ }^{\circ} \mathrm{C}$. In all three plots the solid line corresponds to data - either calculated or experimental and dashed lines to deconvoluted peaks.

can be deconvoluted into two overlapping peaks (Fig. 2c) at $1.14 \AA^{-1}$ and $1.49 \AA^{-1}$. This is behaviour is observed not only in the $\mathrm{N}_{\mathrm{S}}$ phase, but also in the nematic and isotropic liquid. If both wide-angle peaks originate from a single molecule then the analysis presented in eqn (1)-(3) can still be used, whereas if one of the peaks originates from local phase biaxiality in the $\mathrm{N}_{\mathrm{S}}$ phase then this approach may no longer be valid. That the two wide angle peaks are observed in the isotropic liquid and both nematic phases are strong indicators that they do not originate from biaxial local order, however we sought further demonstration that these originate from the molecular shape of RM734 by calculating the molecular form factor (Fig. 2a) and scattered intensity profile (Fig. 2b). The molecular form factor of RM734 shows two diffuse features whose scattering vectors (1.15 $\AA^{-1}$ and $1.44 \AA^{-1}$ ) match both calculated isotropic wideangle scattering data (Fig. 2b) and experimental scattering data in the isotropic liquid (Fig. 2c). Plots of intensity versus $Q$ for the nematic and $\mathrm{N}_{\mathrm{S}}$ phases are given in the ESI, $\dagger$ (Fig. SI2); these show that the position of the deconvoluted peaks does not vary in the different phases (Iso, $\mathrm{N}, \mathrm{N}_{\mathrm{S}}$ ), which is to be expected given their origin in molecular shape.

Having established the origin of the two wide angle peaks, we next measured the orientational order parameters of RM734 as a function of temperature across the nematic and $\mathrm{N}_{\mathrm{S}}$ phases. As shown in Fig. 3, both $\left\langle P_{2}\right\rangle$ and $\left\langle P_{4}\right\rangle$ increase across the nematic phase, reaching maximum values of $\sim 0.55$ and $\sim 0.15$, respectively. At the $\mathrm{N}_{\mathrm{S}}-\mathrm{N}$ transition there is a jump in both $\left\langle P_{2}\right\rangle$ 


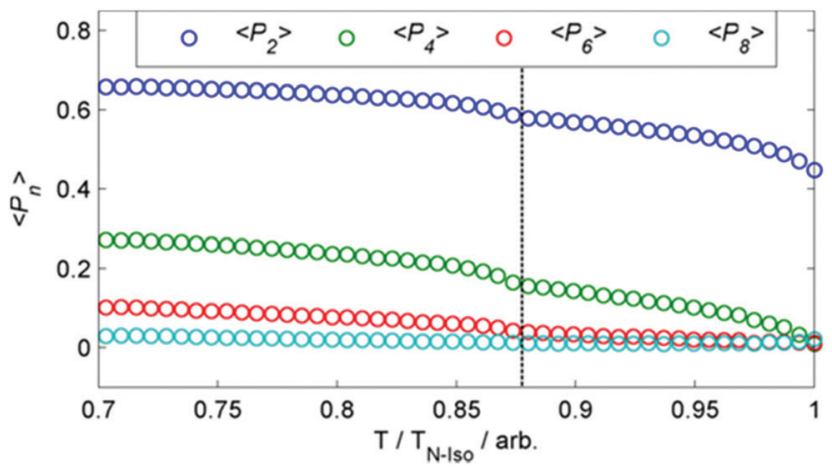

Fig. 3 Plot of the first four even order parameters $\left\langle P_{n}\right\rangle$ as a function of reduced temperature for $\mathrm{RM} 734$. We omit $\left\langle P_{10}\right\rangle$ from this plot as it is effectively zero at all temperatures. The dashed line corresponds to the location of the $\mathrm{N}_{\mathrm{S}}-\mathrm{N}$ phase transition.

and $\left\langle P_{4}\right\rangle$; in the splay nematic phase we find values in the range $\sim 0.60-0.66$ and $\sim 0.20-0.27$ for $\left\langle P_{2}\right\rangle$ and $\left\langle P_{4}\right\rangle$, respectively. Values of $\left\langle P_{6}\right\rangle$ follow the same trend as $\left\langle P_{2}\right\rangle$ and $\left\langle P_{4}\right\rangle$, being in the range $0.00-0.04$ in the nematic phase, and 0.05-0.10 in the $\mathrm{N}_{\mathrm{S}}$ phase. We also determined the higher order parameters $\left\langle P_{8}\right\rangle$ and $\left\langle P_{10}\right\rangle$; the former is zero in the nematic phase and increases slightly to take a maximum value of $\sim 0.03$ in the $\mathrm{N}_{S}$ phase, whereas the latter is effectively zero at all temperatures studied. We note that at the $\mathrm{N}_{\mathrm{S}}-\mathrm{N}$ phase transition there is a clear discontinuity in the first three order parameters, which is to be expected due to the first order nature of this phase transition.

Plotting $\left\langle P_{2}\right\rangle$ versus $\left\langle P_{2}\right\rangle$ allows us to remove the explicit temperature dependence of the two principal order parameters, as shown in Fig. 4. Empty data points correspond to RM734 in the nematic phase; filled data points correspond to the splaynematic phase of the same material. This shows that the relationship between $\left\langle P_{2}\right\rangle$ and $\left\langle P_{4}\right\rangle$ is the same in the nematic and $\mathrm{N}_{\mathrm{S}}$ phases of RM734, with values simply being larger in the

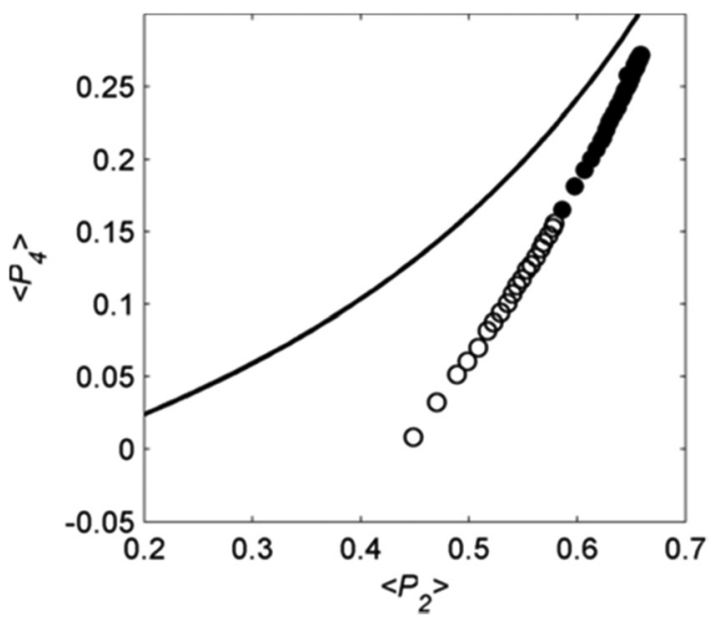

Fig. 4 The variation of $\left\langle P_{4}\right\rangle$ as a function of $\left\langle P_{2}\right\rangle$ for RM734: empty data points correspond to values in the nematic phase $\left(>132.7^{\circ} \mathrm{C}\right)$, filled data points correspond to values in the splay nematic phase $\left(<132.7{ }^{\circ} \mathrm{C}\right)$. Predictions from Maier-Saupe theory (solid line) are given for comparison. lower temperature nematic phase. Experimental orientational order parameters were compared with Maier-Saupe (MS) theory, presented as a solid black line. It is immediately apparent that, at a given value of $\left\langle P_{2}\right\rangle$ the corresponding value of $\left\langle P_{4}\right\rangle$ is smaller than is predicted by either MS theory. It should be noted that MS theory is concerned with predicting the orientational properties of uniaxial nematic liquid crystals and so it is therefore not surprising that it fails to describe the orientational properties of the splay modulated nematic phase, however, this does highlight the need for an analytic theory of this phase of matter to be developed.

From the first five even orientational order parameters we reconstruct the orientational distribution function (ODF) across the temperature range studied (Fig. 5a). Truncating the reconstruction of the ODF at the 5 th $\left\langle P_{2 n}\right\rangle$ term (ESI, $\dagger$ eqn (S12)) or the sixth $f_{2 n}$ term (ESI, $\dagger$ eqn (S13)) gave essentially identical results. The use of higher order parameters (i.e. $\left.\left\langle P_{8}\right\rangle,\left\langle P_{10}\right\rangle\right)$ allows finer detail to be resolved in the ODF which may otherwise be lost through truncation effects. In both the nematic and splay nematic phases the ODF is (to a first approximation) Gaussian with its maxima at $\beta=0^{\circ}$, indicating that in terms of orientational order the $\mathrm{N}_{\mathrm{S}}$ phase is locally similar to the preceding nematic (Fig. 5b). As would be expected, we find the FWHM of the ODF decreases as a function of temperature, with a small discontinuity at the $\mathrm{N}_{\mathrm{S}}-\mathrm{N}$ transition (Fig. $5 \mathrm{c}$ ). The change in orientation from one molecule to its nearest neighbours that results from the splay modulation is therefore small enough that it is not observable
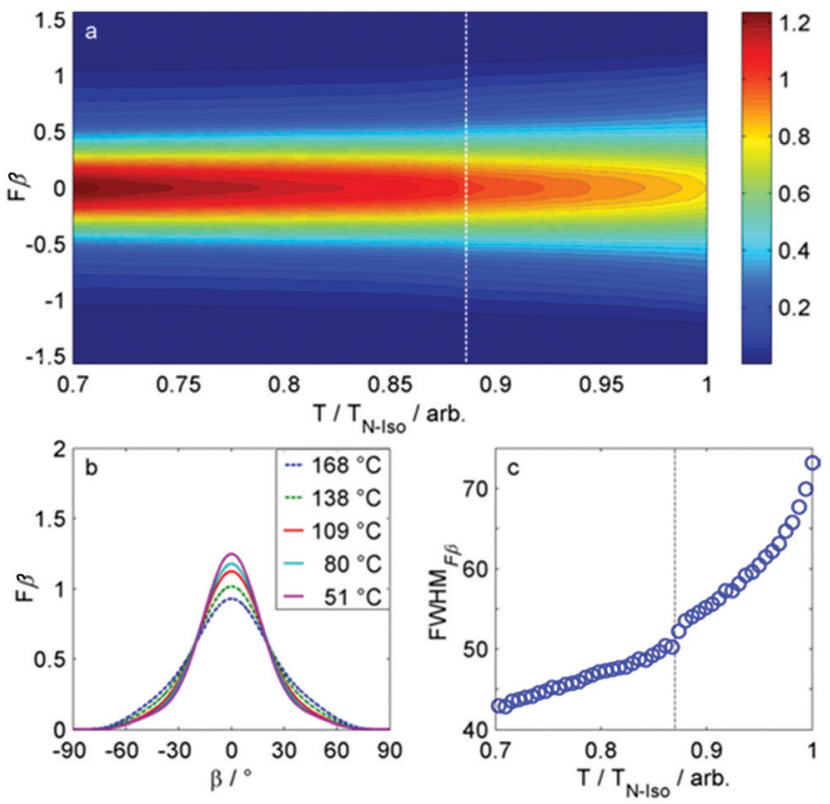

Fig. 5 (a) Contour plot of the orientational distribution function (ODF) as a function of reduced temperature, where the white dashed line indicates the $\mathrm{N}_{\mathrm{S}}-\mathrm{N}$ transition temperature. (b) Plot of the ODF of RM734 at five temperatures - dashed lines correspond to the nematic phase, solid lines correspond to the splay-nematic phase. (c) Plot of the HWHM of the ODF as a function of reduced temperature - the dashed line corresponds to the $\mathrm{N}_{\mathrm{S}}-\mathrm{N}$ phase transition. 
by WAXS. The implication is that the periodicity of the $\mathrm{N}_{\mathrm{S}}$ phase is large rather than being of the order of a few nanometers as is the case with the $\mathrm{N}_{\mathrm{TB}}$ phase.

We also studied the orientational order of an analogue of RM734 used in this work in which the nitro group is replaced with a nitrile, in this manuscript we refer to this material as 'RM734-CN' and it has the phase sequence Cr 173.2 N 200.4 Iso. RM734-CN does not exhibit the splay-nematic phase, the temperature dependence of the first four even $\left\langle P_{n}\right\rangle$ order parameters is typical of that of a nematic phase, and the discontinuity observed at the $\mathrm{N}_{\mathrm{S}}-\mathrm{N}$ transition for RM734 is absent. Further discussion and data are given in the ESI. $\dagger$ The behaviour of the orientational order parameters at the $\mathrm{N}_{\mathrm{S}}-\mathrm{N}$ transition appears to be unique, and is distinct from other presently known modulated nematic phases. ${ }^{7,8}$ Measurement of the orientational order parameters by WAXS - coupled with the absence of Bragg scattering in small-angle X-ray scattering (SAXS) to confirm a positionally disordered phase could be considered diagnostic of the splay nematic phase, and therefore provides a means to identify of this phase of matter.

\section{Conclusions}

Wide-angle X-ray scattering experiments have been used to probe the orientational order in the nematic $(\mathrm{N})$ and splay nematic $\left(\mathrm{N}_{\mathrm{S}}\right)$ phases of the liquid crystalline material RM734. The $\mathrm{N}_{\mathrm{S}}$ phase displays a slightly greater degree of orientational order than the classical nematic phase, with a discontinuous increase in the first four even $\left\langle P_{n}\right\rangle$ order parameters at the $\mathrm{N}_{\mathrm{S}}-\mathrm{N}$ phase transition, whereas $\left\langle P_{10}\right\rangle$ was found to be effectively zero at all temperatures. Reconstruction of the orientational distribution function (ODF), and the lack of off axis correlation peaks indicates that the change in orientation between two neighbouring molecules that results from the splay modulation of the $\mathrm{N}_{\mathrm{S}}$ phase is small, too small to be measured by WAXS. This suggests that the periodicity of the splay-nematic phase, at least in RM734, is larger than the few tens of nanometers envisioned originally. ${ }^{11}$

\section{Conflicts of interest}

There are no conflicts to declare.

\section{Acknowledgements}

The EPSRC is thanked for funding the Bruker D8 SAXS/WAXS equipment used in this work via grant EP/K039660/1. AM acknowledges the financial support from the Slovenian Research Agency (research core funding No. P1-0192).

\section{Notes and references}

1 I. Dozov, Europhys. Lett., 2001, 56, 247-253.

2 M. Cestari, S. Diez-Berart, D. A. Dunmur, A. Ferrarini, M. R. de la Fuente, D. J. Jackson, D. O. Lopez, G. R. Luckhurst, M. A. Perez-Jubindo, R. M. Richardson, J. Salud, B. A. Timimi and H. Zimmermann, Phys. Rev. E: Stat., Nonlinear, Soft Matter Phys., 2011, 84, 031704.

3 D. Chen, M. Nakata, R. Shao, M. R. Tuchband, M. Shuai, U. Baumeister, W. Weissflog, D. M. Walba, M. A. Glaser, J. E. Maclennan and N. A. Clark, Phys. Rev. E: Stat., Nonlinear, Soft Matter Phys., 2014, 89, 022506.

4 R. J. Mandle, Soft Matter, 2016, 12, 7883-7901.

5 A. Jakli, O. D. Lavrentovich and J. V. Selinger, Rev. Mod. Phys., 2018, 90, 045004.

6 C. Zhu, M. R. Tuchband, A. Young, M. Shuai, A. Scarbrough, D. M. Walba, J. E. Maclennan, C. Wang, A. Hexemer and N. A. Clark, Phys. Rev. Lett., 2016, 116, 147803.

7 G. Singh, J. X. Fu, D. M. Agra-Kooijman, J. K. Song, M. R. Vengatesan, M. Srinivasarao, M. R. Fisch and S. Kumar, Phys. Rev. E, 2016, 92, 060701.

8 R. J. Mandle and J. W. Goodby, Phys. Chem. Chem. Phys., 2019, 21, 6839-6843.

9 R. J. Mandle, S. J. Cowling and J. W. Goodby, Phys. Chem. Chem. Phys., 2017, 19, 11429-11435.

10 R. J. Mandle, S. J. Cowling and J. W. Goodby, Chemistry, 2017, 23, 14554-14562.

11 A. Mertelj, L. Cmok, N. Sebastian, R. J. Mandle, R. R. Parker, A. C. Whitwood, J. W. Goodby and M. Copic, Phys. Rev. X, 2018, 8, 041025.

12 H. Pleiner and H. R. Brand, Europhys. Lett., 1989, 9, 243-249.

13 N. Chaturvedi and R. D. Kamien, eprint arXiv:1904.09210, 2019, arXiv:1904.09210.

14 H. Nishikawa, K. Shiroshita, H. Higuchi, Y. Okumura, Y. Haseba, S. I. Yamamoto, K. Sago and H. Kikuchi, Adv. Mater., 2017, 29, 1702354.

15 G. Barbero and I. Lelidis, Liq. Cryst., 2019, 46, 535-542.

16 M. T. Sims, L. C. Abbott, R. M. Richardson, J. W. Goodby and J. N. Moore, Liq. Cryst., 2018, 46, 11-24.

17 D. M. Agra-Kooijman, M. R. Fisch and S. Kumar, Liq. Cryst., 2018, 45, 680-686.

18 P. V. Konarev, V. V. Volkov, A. V. Sokolova, M. H. J. Koch and D. I. Svergun, J. Appl. Crystallogr., 2003, 36, 1277-1282.

19 D. Franke, M. V. Petoukhov, P. V. Konarev, A. Panjkovich, A. Tuukkanen, H. D. T. Mertens, A. G. Kikhney, N. R. Hajizadeh, J. M. Franklin, C. M. Jeffries and D. I. Svergun, J. Appl. Crystallogr., 2017, 50, 1212-1225.

20 D. Svergun, C. Barberato and M. H. J. Koch, J. Appl. Crystallogr., 1995, 28, 768-773. 